\title{
NOTE ON THE EXISTENCE OF REISSNER'S FIBRE IN HIGHER VERTEBRATES.
}

By SIR VICTOR HORSLEY, F.R.S., F.R.C.S.

(From the Laboratory of Chemical Pathology, University College, London.)

Morphotogical study of the central nervous system has for a number of years been notably advanced on the interesting structure known as Reissner's fibre by the work of Porter E. Sargent, who has shown that in the teleostean it arises in the torus longitudinalis tecti, extending thence caudally along the central canal of the nervous system, and that it is probably present in all classes of vertebrates.

For a clear survey of the literature of the subject we are also indebted to Sargent, to whose papers [3], [4], [5] reference must be made for his discussion of the character of the fibre and its anatomical structure.

The importance of its relation to the habenular region having recently formed the subject of a communication by Professor Dendy suggested to me searching the various series of sections of apes' brains in my possession as a comparison with the lower vertebrates.

The physical character of the fibre, well described by Sargent, viz., its stiff elastic recoil, causes it to be readily lost in preparing sections of the central canal of the nervous system. Hence, I have been able to find it preserved in good lengths in one animal only, Macacus cynomolgus, in a series of sagittal sections stained by Marchi's method, cut especially thick; in this case a considerable clot in the central canal entangled the fibre and partly secured it in situ.

It is, however, not only the evidence of the occurrence of this interesting structure in the higher apes which leads me to publish this note, but also that the further question of the function of the fibre deserves closer attention from neurologists.

Naturally, the greatest deference is due to the.opinion of Sargent, 


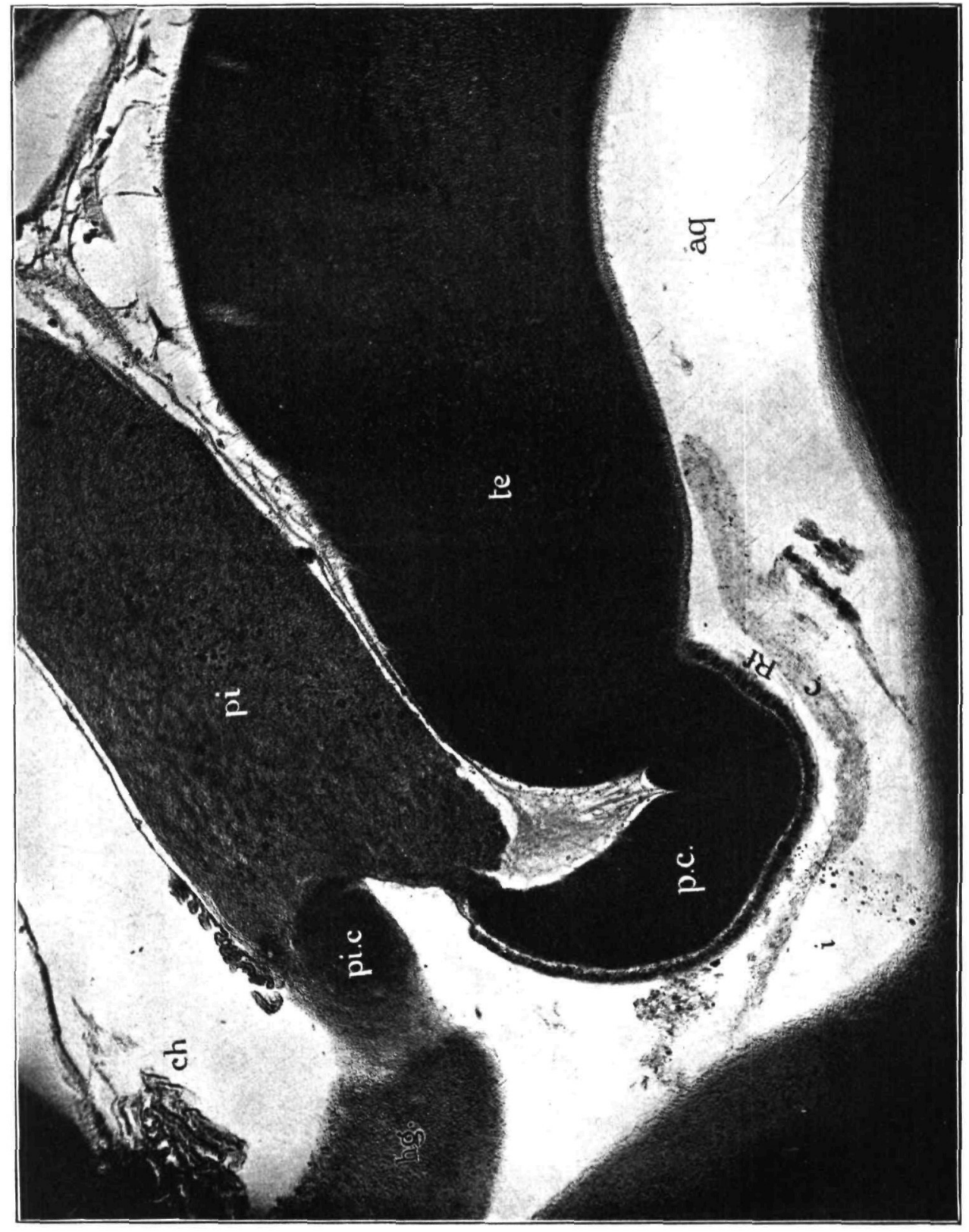

Erg. 1.

Macacus cynomolgus. Longitudinal section. ch, choroid plexus; hg., mesial surface of ganglion habenule; pi.c, commissura habenularis; pi, corpus pinealis; p.c., commissure posterior; te, tectum; i, iter; c, clot; Rf, letters placed at the broken end of Reissner's fibra, which extends forwards beneath the columnar epithelium as a wavy line into the clot; aq. aqueductus Fallopii. 


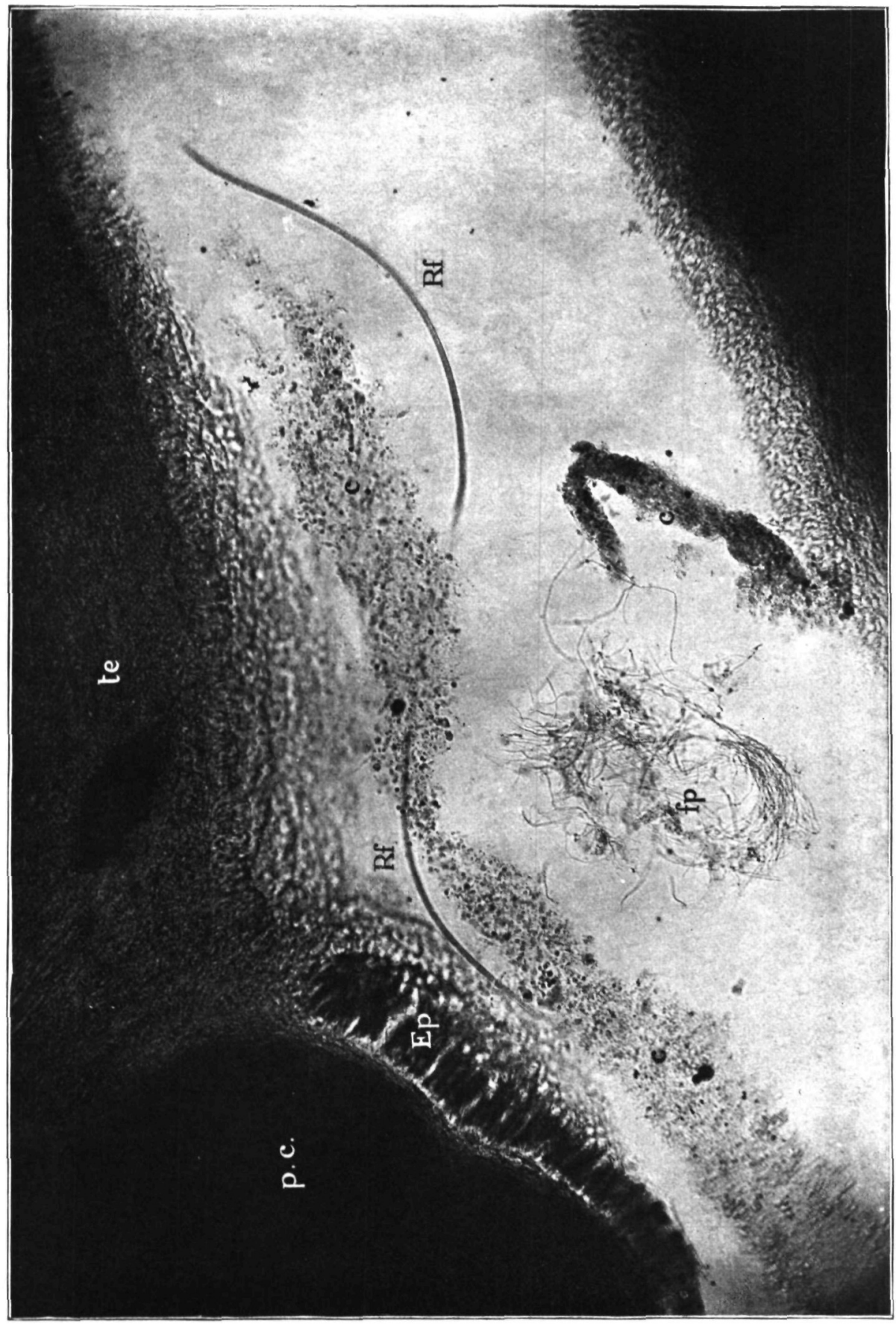

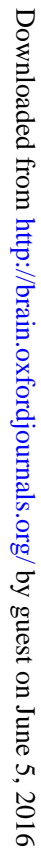

FIG. 2.

Macacus cynomolgus. Next section in the series to fig. 1. Reissner's fibre, Rf, entangled in the clot, c, continues into the aqueduct. Compare for artefacts the clots, $c$, $c$, and the filter paper fibres, f p. E p, ventral columnar epitbelium (Dendy [1]). 


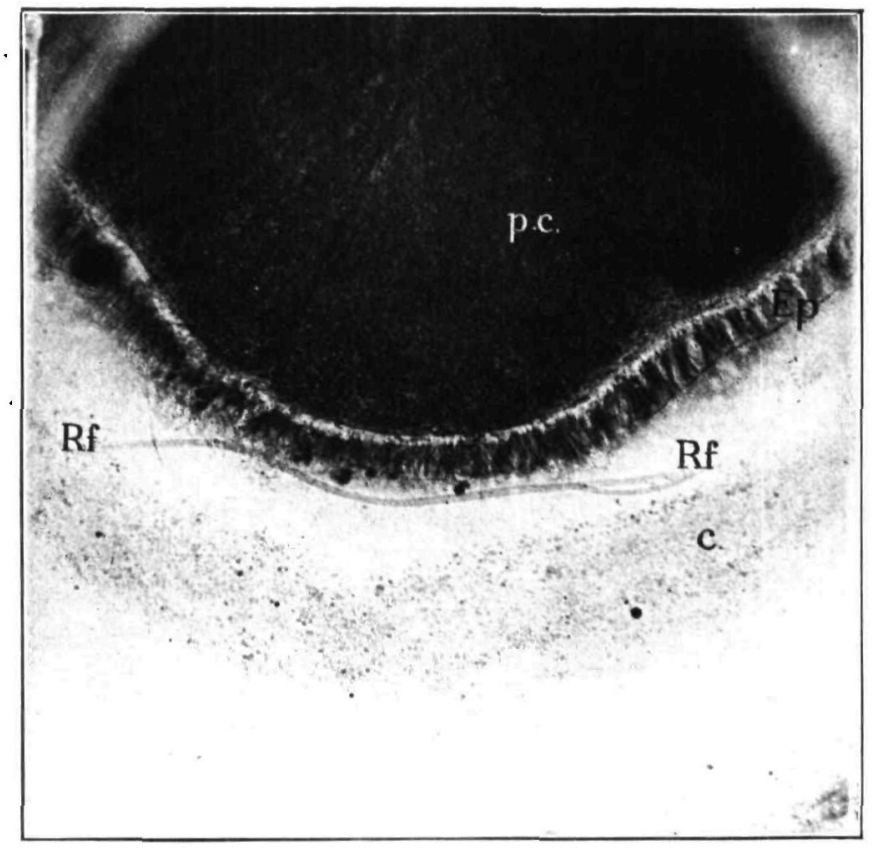

Fig. 3.

Macacus cynomolgus. Posterior lualf of Reissner's fibre, shown in fig. 1, more highly magnified. Lettering as figs, 1 and 2. The elastic recoil of the broken fibre, is characteristically shown at the right hand end.

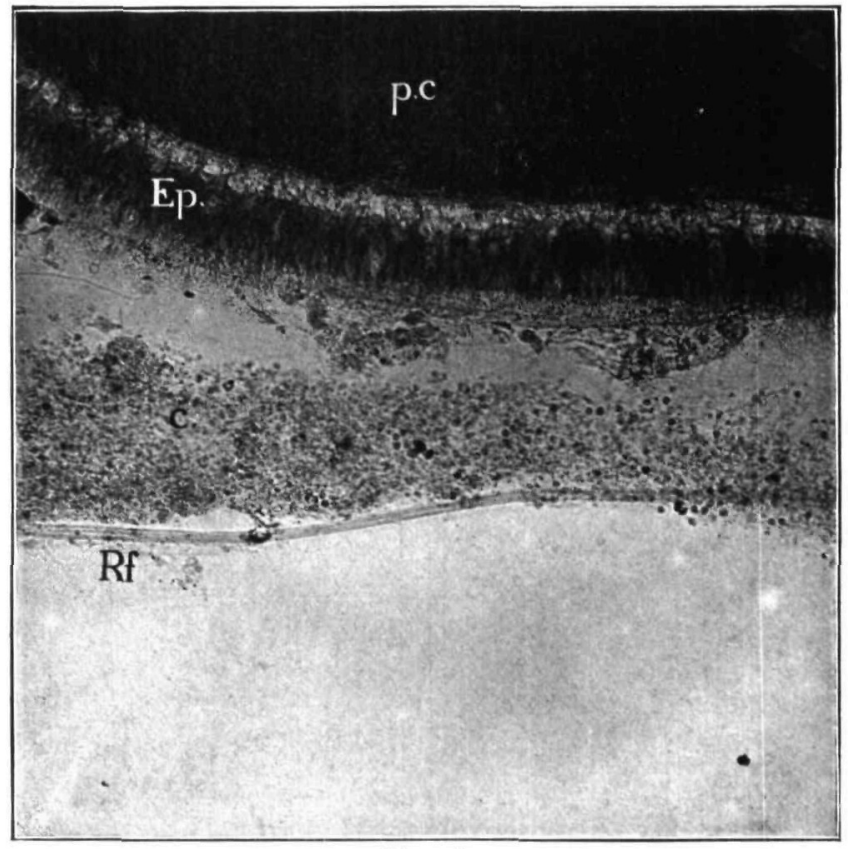

Fig. 4.

Macacus cynomolgus. The anterior half of Reissner's fibre, as shown in fig. 1 , more highly magnified. The fibre, Rf, shows well (especially under a lens) the appearance of fibrillation or of a core, and reacts in staining more as an epitbelial structure than a nerve fibre. 
who has essentially made this subject his own; but even the study of his extremely interesting papers (especially [5]) does not produce

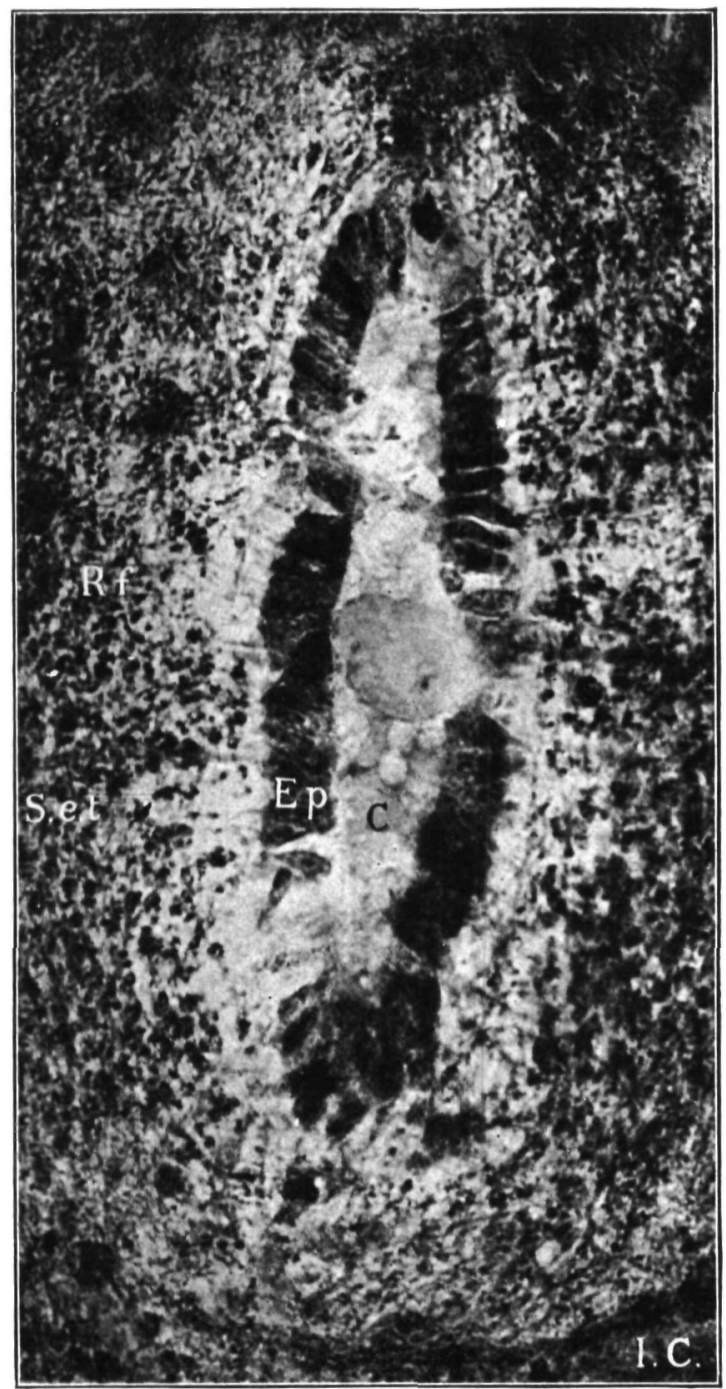

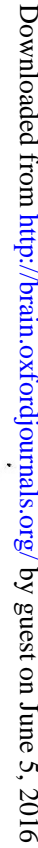

Fia. 5.

Macacus rhesus. Transverse section of spinal cord at the level of the first cervical segment highly magnified. The centre of the central canal is occupied by Reissner's fibre, the rest by altered blood-clot, C. The fibre shows a spot suggesting the existence of a core. The section has been lightly compressed. E p, epithelium.

conviction that the fibre is in fact an internuncial nerve tract which rivals or even exceeds in importance the tecto-spinal system, rather 
than a skeletal or ependymal structure. On this point, namely, the physiological function of Reissner's fibre, I venture to think Sherrington [6] has accepted somewhat prematurely the proofs that at present are before us of its being in the higher mammal an optic reflex nerve path.

As Sargent has abundantly established, Reissner's fibre begins by several or many roots of origin from a wide stretch of the ventral

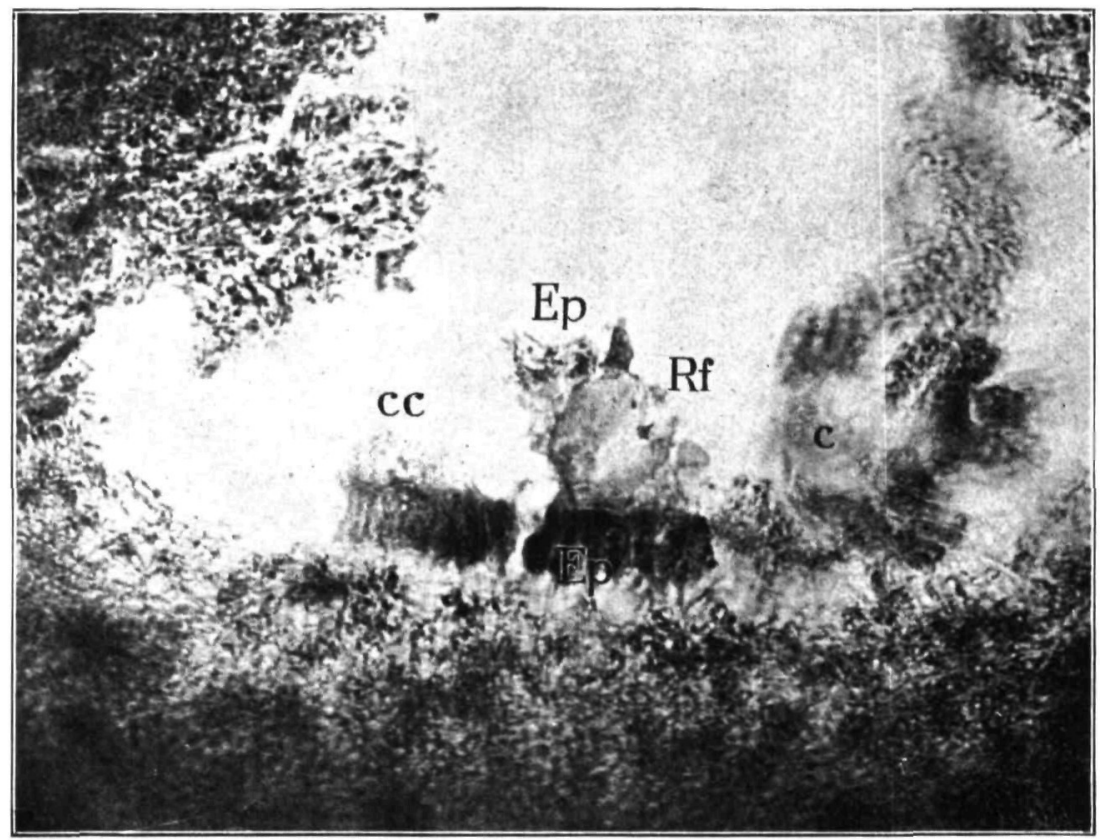

FIG. 6.

Macacus rhesus. A section close to that shown in fig. 5 dissociated by pressure, showing Reissner's fibre crushed, Rf. Its structure is seen to be unlike that of a nerve fibre, and the condensed peripheral border does not stain like myelin with the osmic acid. Ep, epithelium of central canal ; cc, canalis centralis.

(ventricular) aspect of the tectum in the posterior commissural and habenular region. For its origin in the frog see [5]. He has shown that its attachments to the roof of the aqueduct extend from the habenular ganglion in front to the recessus mesocœli behind the caudal border of the posterior commissure. Here it arises.in fish from the 


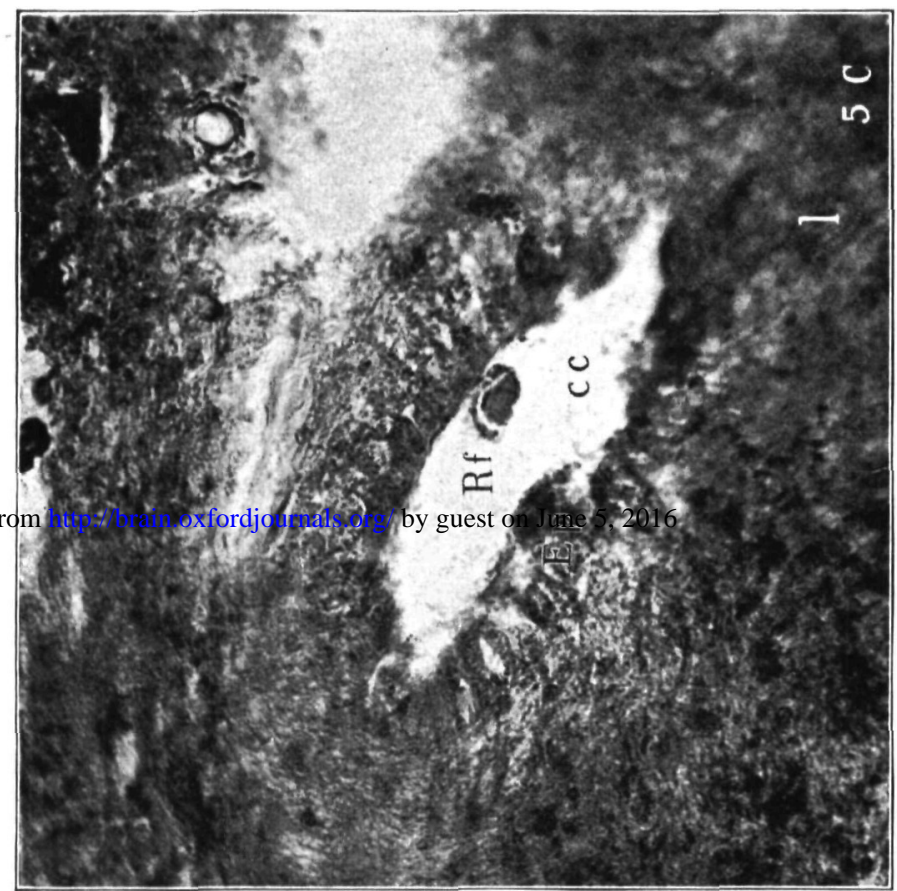

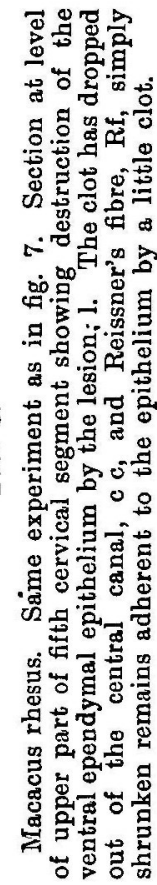

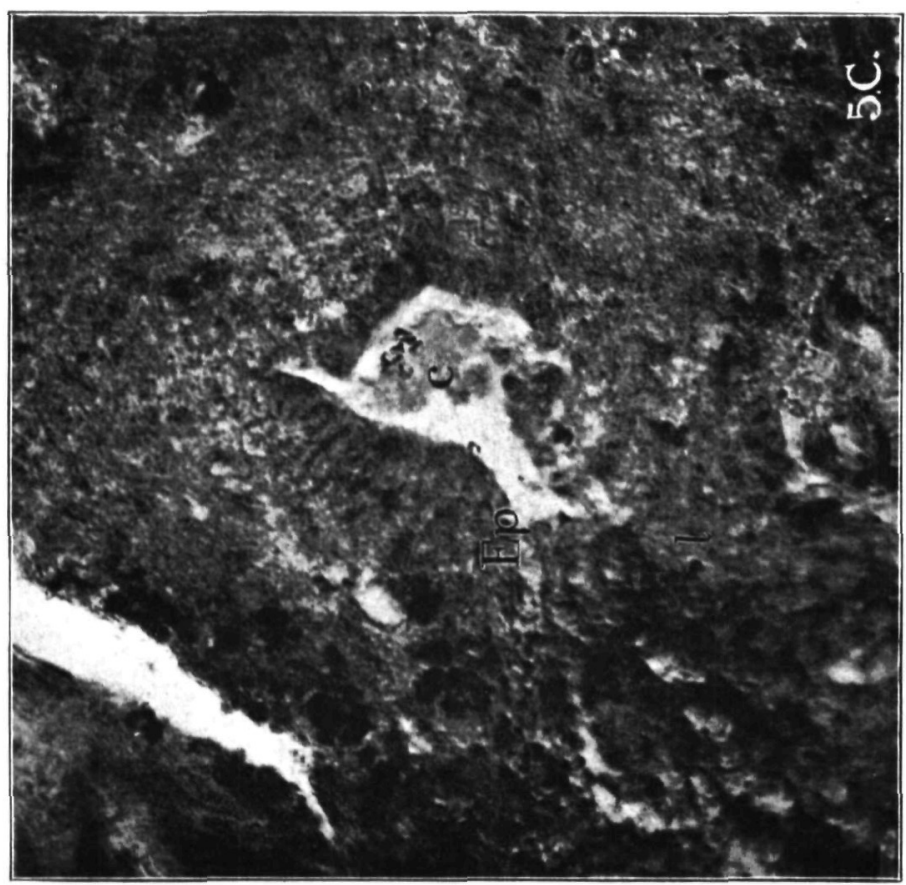

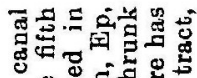
ङ

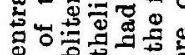

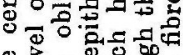
岕.

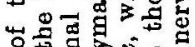

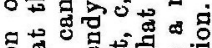

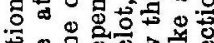

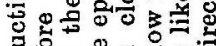

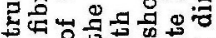

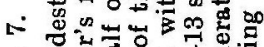
- 丁

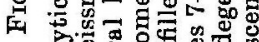

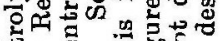

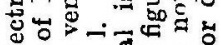

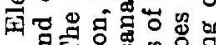

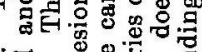

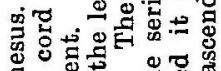

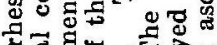

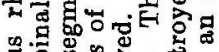

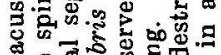

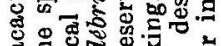

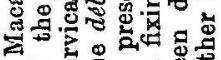

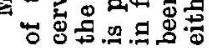


torus longitudinalis, and especially from the nucleus of large corpuscles (nidulus tectalis, Sargent), which has received various names, including the unfortunate expression roof nucleus. The fibre composed by the fusion of its roots of origin extends as a single structure down the iter, through the fourth ventricle and throughout the central canal of the spinal cord, giving off branches to the wall of the latter tube. The

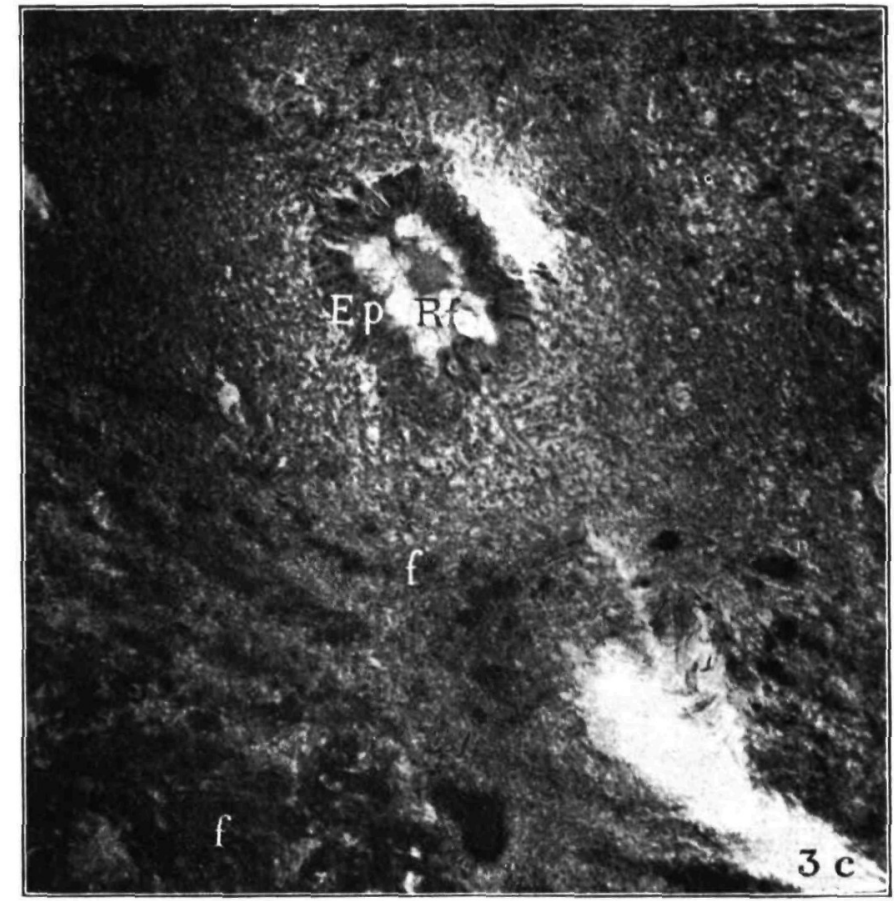

Fig. 9.

Mncacus rhesus. Same experiment as in fig. 7. Section at level of third cervical segment. Tho central canal shows still much injury to the epithelium, E p, while Resssner's fibre, Rf, only shows shrinkage. Nerve fibres showing myelin staining are seen at f, $f$.

structure of the fibre is regarded by Sargent as consisting of axones covered by a myelin sheath.

That the interior of the fibre often appears to be fibrillar is obvious, and is shown in figs. 1 to 4 (on examination with a lens), but neither the texture nor the appearance of the fibre resembles any nerve tract. Especially is this noticeable in the stiff resiliency of its structure, which 
is more characteristic of an almost chitinous or skeletal structure than that of a soft nerve fibre (see also figs. 5 and 6 ).

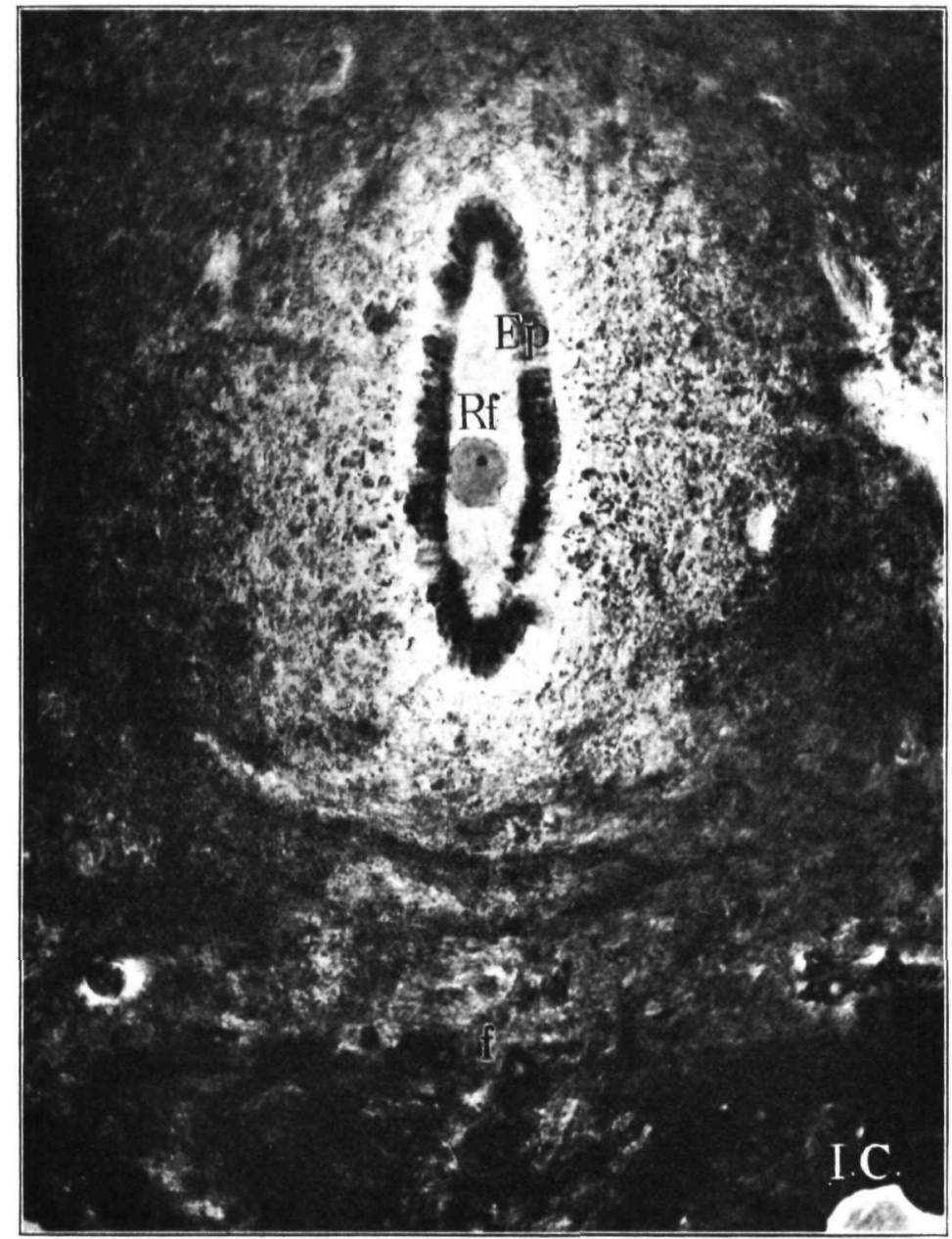

Fig. 10.

Macacus rhesus. Same experiment as in fig. 7. Section at level of first corvical segmont. Reissuer's fibre, Rf, is well secn in the canal, exhibiting its somewhat condensed periphery and the appearance of a core (not constant). Compare the diameter of the fibre in this figure with that in fig. 13 .

As regards the existence of a myelin sheath, it is evident from the photographs that the osmic acid in fact stains the outer, or 
indeed any part of the fibre, less intensely than the normal nerve fibres, and the same is observable in other animals (Macacus rhesus; three individuals).

Moreover none of the figures given by the various authors writing on this structure appear to me to confirm the view that the outer part of the fibre is a medullary sheath, and this point, as well as the

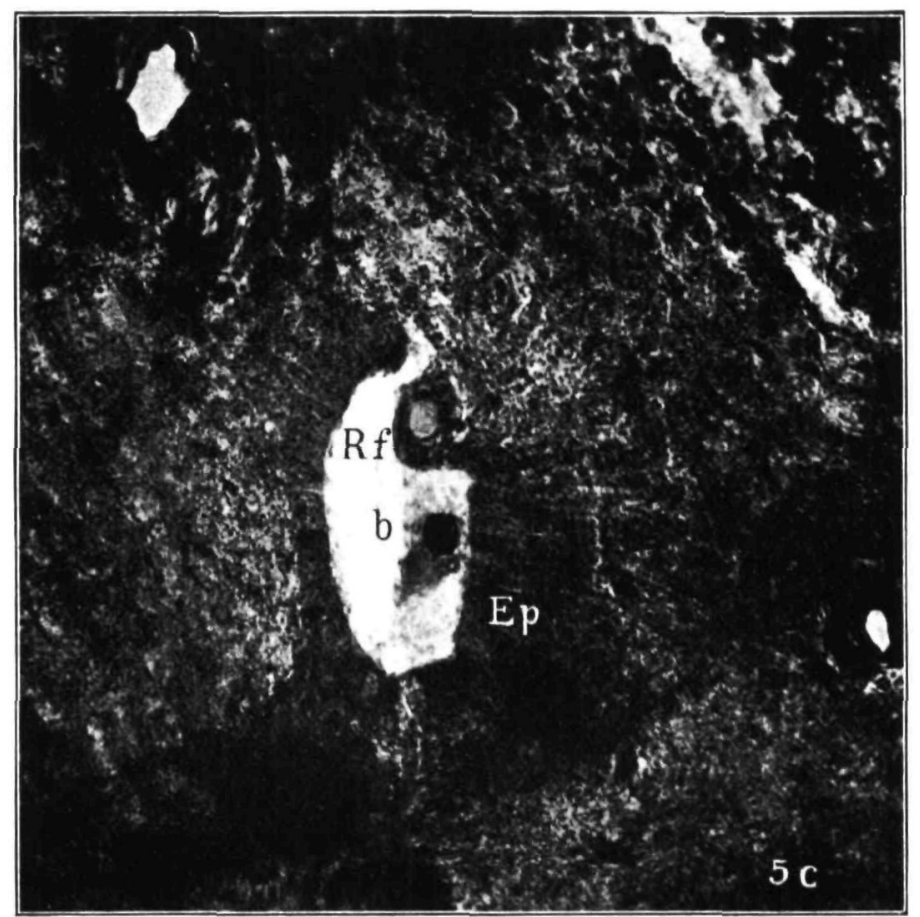

Fig. 11.

Macacus rhesus. Same experiment as in fig. 7. Section at level of lower border of fifth cervical segment. The epithelium of the central canal has been slightly injured over ove-sixth of its circumference. Reissner's fibre, $R \mathrm{f}$, is buried in the swollen epithelium. At b is a blood-clot, the hæmoglobin content of four or five red corpuscles being fused into the black mass in the centre. Reissner's fibre is unaltered, i.e., not " degenerated."

central connection and origin of the fibre, must receive reinvestigation, at any rate before we can assume that the fibre is in the higher vertebrate more than a residual structure.

Finally, I have recently obtained evidence which appears to me to be inexplicable if the fibre is to be regarded as a nerve bundle. This is the 
crucial test of degeneration. In a research which I am carrying on with Dr. McNalty, and in which I have made minute electrolytic lesions in the interior of the spinal cord, causing clots in the central canal and so fixing the fibre in situ, the following result has been obtained in a Macacus rhesus. The lesion destroyed in this instance the fibre at the

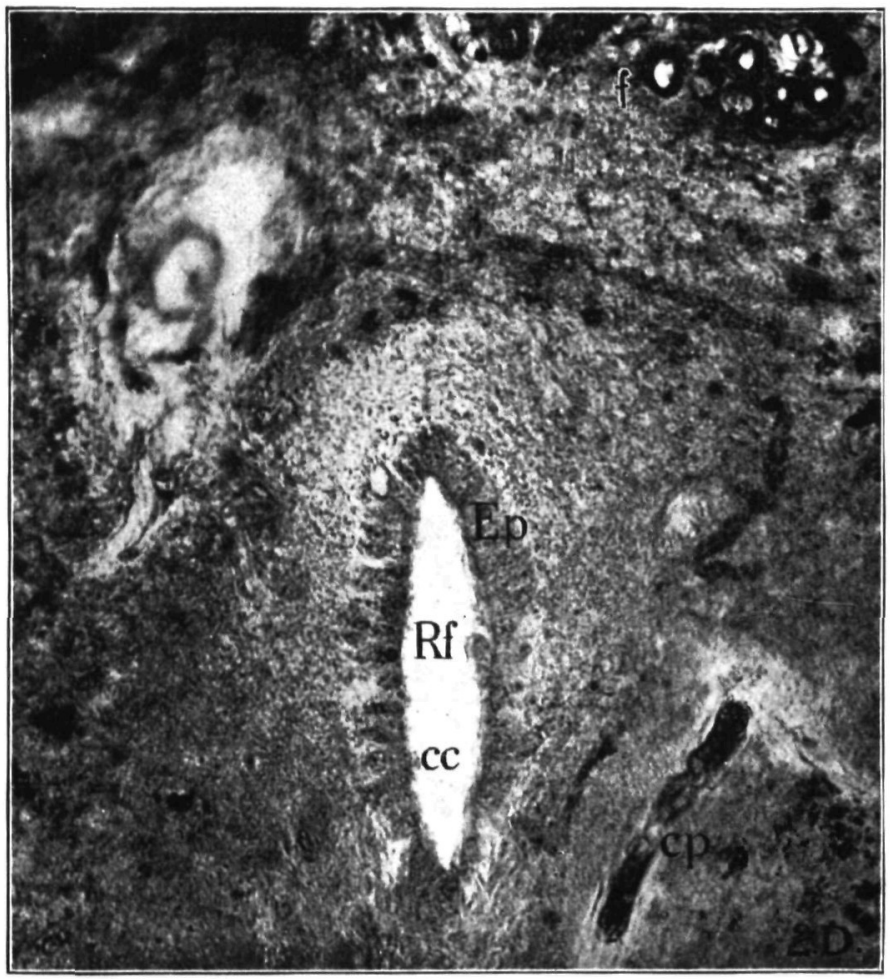

FIG. 12.

Macacus rhesus. Same experiment as fig. 7. Section at level of the second dorsal segment. Reissner's fibre, Rf, of normal appearance, lies between $\mathrm{Rf}$ and the ependymal epithelium. f, normal nerve fibres deeply stained with the osmic acid; $c p$ capillary blood-vessel ; cc, canalis centralis.

level of the fifth cervical segment (fig. 7), but neither upwards nor downwards from this point does the fibre exhibit any degenerative or abnormal changes, except that of moderate shrinkage from the fifth cervical to the third cervical segment-parallel, in fact, to the longitudinal extent of the electrolytic lesion (see figs. 7 to 13 ). 


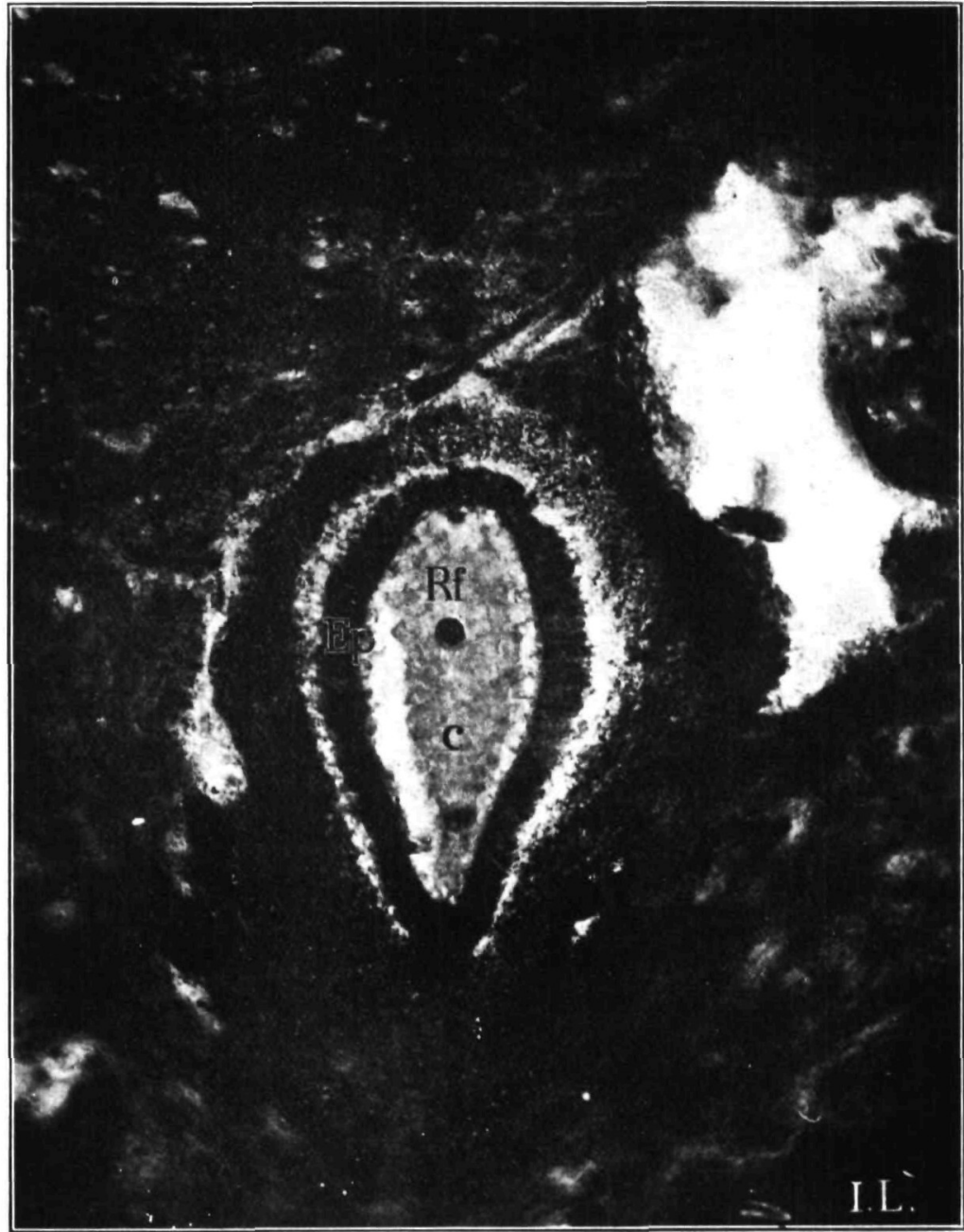

Fra. 13.

Macacus rhesus, Same experiment as fig. 7. Section at level of the first lumbar segment. The central canal is filled with clot, c. Reissner's fibre, normal in appearance and size, is seen below Rf. The tapering of the fibre (Sargent) from the first cervical (fig. 10) to the first lumbar segment is well shown in this series. Ep, epithelium.

\section{SUMMARY.}

(1) Reissner's fibre is present and well marked in Macacus cynomolgus and rhesus.

(2) Reissner's fibre in its normal structure and in its not reacting to injury does not resemble a nerve fibre or tract. 


\section{Literature.}

[1] Dendy. Proc. Roy. Soc., vol. 1xix., p. 485; Science Progress, No. 6, 1907.

[2] Nicholcs. Nature, February 13, 1908, p. 344.

[3] Sargent. Anatom. Anzeig, 1900, Bd. xvii., S. 32 (for literature and general course of the fibre).

[4] Tbid. "Mark Anniversary Volume," New York, 1903, p. 399 (for description of the torus longitudinalis and origin of the fibre).

[5] Ibid. Bull. Mus. Comp. Zool. Harv. Coll., 1904, vol. xlv., No. 3, p. 129 (principal paper; full title: "The Optic Reflex Apparatus of Vertebrates for Short Circuit transmission of Motor Reflexes through Reissuer's Fibre, its Morphology, Ontogeny, Phylogeny and Function.-Part I. The Fish-like Vertebrates.")

[6] Sherrington. "The Integrative Action of the Nervous System," 1906, p. 329. 